\title{
When No Reason For Is A Reason Against
}

\author{
Benjamin Eva* Stephan Hartmann ${ }^{\dagger}$
}

June 29, 2017

So far I have not seen a good argument for the existence of God. This observation is, in turn, a good argument against the existence of God. (Hanson (1971))

\begin{abstract}
We provide a Bayesian justification of the idea that, under certain conditions, the absence of an argument in favour of the truth of a hypothesis $\mathrm{H}$ constitutes a good argument against the truth of $\mathrm{H}$.
\end{abstract}

\section{Introduction}

Consider the following familiar argument schemes:

- There is no good argument for the existence of God, so I am an atheist.

- You can't prove that my client is guilty, so I believe that she is innocent.

- There is no good reason to think that leaving the fan on overnight is dangerous, so I'll leave it on.

Although arguments of this type are commonly used in a wide range of reasoning contexts, it is far from clear how they can be accounted for in

*Munich Center for Mathematical Philosophy, LMU Munich, 80539 Munich (Germany) - http://www.mcmp.philosophie.uni-muenchen.de/people/faculty/eva_benjamin/index.html benedgareva@icloud.com.

${ }^{\dagger}$ Munich Center for Mathematical Philosophy, LMU Munich, 80539 Munich (Germany) http://www.stephanhartmann.org - s.hartmann@lmu.de. 
the context of standard Bayesian epistemology. For, there is no canonical mechanism for updating on the absence of relevant evidence. In this article, we show that these arguments are structurally isomorphic to the "no alternatives arguments' recently analysed by Dawid, Hartmann and Sprenger (2015), and so can, in certain circumstances, be given a fully Bayesian vindication in the same way.

\section{No Alternatives}

According to the traditional Bayesian paradigm, scientific theories are assessed in the light of relevant empirical data (see, e.g., Howson and Urbach (2005)). In recent years there has been much debate concerning how theories can be evaluated in the absence of such data. In particular, it is well known that some theories of contemporary fundamental physics are extremely far from being empirically testable (Dawid (2013)). This situation motivates the idea that theories can also be assessed in the absence of empirical data. Towards this end, Dawid, Hartmann and Sprenger (2015) (henceforth DHS) consider the 'no alternatives argument' (NAA), which has the following form:

P1: Theory or hypothesis $\mathrm{H}$ satisfies several desirable conditions.

P2: Despite a lot of effort, the scientific community has not yet found an alternative to $\mathrm{H}$ that also satisfies these conditions.

$\mathrm{C}$ : Hence we have one good reason in favor of $\mathrm{H}$.

DHS note that NAA type arguments play a prominent role in contemporary physics, and so should be taken seriously (though not uncritically accepted without further inspection) by students of scientific epistemology. Crucially for our purposes, they also show that these arguments can be faithfully represented in a Bayesian setting, once one countenances the possibility of non-empirical evidence. ${ }^{1}$

\footnotetext{
${ }^{1}$ We submit that the term "non-empirical evidence" might be misleading. A better term is perhaps "indirect evidence" which is evidence that does not follow deductively or inductively from the hypothesis under consideration. Clearly P2 represents empirical evidence, but it is indirect as the failure of the scientific community to come up with an alternative is typically not a deductive consequence of the hypothesis in question.
} 
Let $\mathrm{F}$ be the proposition 'the scientific community has not yet found an alternative to the hypothesis H'. The idea is to show that $\mathrm{F}$ confirms $\mathrm{H}$, i.e. that $P(\mathrm{H} \mid \mathrm{F})>P(\mathrm{H})$, where $P$ is the subjective probability distribution of some agent. Furthermore, let $Y$ be a propositional variable that has the values $\mathrm{Y}_{\mathrm{i}}$ for each integer $i \geq 0 .^{2}$ We interpret $\mathrm{Y}_{\mathrm{i}}$ as the proposition 'there exist $i$ possible alternatives to $H^{\prime}$. Intuitively, scientists will have prior beliefs about the values of $Y$. For example, it might be that although a scientist has not yet found an alternative to $\mathrm{H}$, she is certain that there is one, and so she will assign $\mathrm{Y}_{0}$ a prior probability of 0 . Generally though, scientists may be uncertain about whether or not alternatives exist, and if so, how many.

Next, it seems plausible that one's prior belief in $\mathrm{H}$ should scale inversely with the number of alternative theories. If there is no alternative to $\mathrm{H}$, that should render $\mathrm{H}$ maximally likely. If however $\mathrm{H}$ is only one of many competing theories, all of which do the job, then $\mathrm{H}$ will be less believable. The probability that $\mathrm{H}$ is true will certainly not go up if more alternatives become available. It also seems reasonable that the more alternatives to $\mathrm{H}$ there are, the more likely the scientific community will be to find one. It will certainly not become less likely that the scientific community finds an alternative if more alternatives become available. Finally, DHS note that knowledge of the value of $Y$ renders $F$ probabilistically independent of $H$ : If a scientist knows that there are, say, five alternatives to $\mathrm{H}$ (even without knowing what these theories are), the fact that the scientific community hasn't found one of them yet doesn't tell us anything new about whether or not $\mathrm{H}$ is true. $\mathrm{F}$ is only relevant to $\mathrm{H}$ when we are uncertain about the value of $Y$. These considerations can be summarised by the following four conditions:

NAA1: $\forall i \geq 0, y_{i}:=P\left(\mathrm{Y}_{\mathrm{i}}\right) \in[0,1)$.

NAA2: $h_{i}:=P\left(\mathrm{H} \mid \mathrm{Y}_{\mathrm{i}}\right)$ are monotonically decreasing in $i$.

NAA3: $f_{i}:=P\left(\mathrm{~F} \mid \mathrm{Y}_{\mathrm{i}}\right)$ are monotonically decreasing in $i$.

NAA4: $H$ and $F$ are conditionally independent on $Y$, i.e. $P\left(\mathrm{H} \mid \mathrm{Y}_{\mathrm{i}}, \mathrm{F}\right)=$ $P\left(\mathrm{H} \mid \mathrm{Y}_{\mathrm{i}}\right)$.

\footnotetext{
${ }^{2}$ Note that we represent propositional variables in italic script and their values in roman script. See Bovens and Hartmann (2003).
} 
Armed with these conditions, DHS prove the following theorem: ${ }^{3}$

Theorem 1 Let $P$ be a probability distribution satisfying the conditions NAA1 - NAA4. Then $\mathrm{F}$ confirms $\mathrm{H}$, i.e. $P(\mathrm{H} \mid \mathrm{F})>P(\mathrm{H})$, if there exists a pair $(i, j)$ with $i>j$ such that (i) $y_{i} y_{j}>0$, (ii) $f_{i}<f_{j}$, and (iii) $h_{i}<h_{j}$.

Note that the justification for the existence of a pair $(i, j)$ satisfying the conditions (i) to (iii) is essentially the same as the motivation for NAA1 to NAA3. Condition (i) requires that we are uncertain about whether there are $i$ or $j$ many alternatives to $\mathrm{H}$. Condition (ii) says that we are more likely to have found an alternative to $\mathrm{H}$ if there exist $i$ alternatives than we are if there exist $j$ alternatives, where $i>j$. Similarly, (iii) says that $\mathrm{H}$ is more likely to be true when there are $j$ alternatives than it is when there are $i$ alternatives, where $i>j$. We only require the existence of one pair $(i, j)$ satisfying these three conditions.

So under some weak and intuitive conditions, the NAA works and scientific theories can be confirmed in this way.

\section{No Reason For Is a Reason Against}

Now consider the proposition H: 'God exists', and suppose that we are uncertain about the truth of $\mathrm{H}$. Let $\mathrm{F}$ be the proposition ' $\mathrm{I}$ have not yet found a good argument in favour of H', and let $Y$ be a propositional variable whose values are the propositions $Y_{i}$ : 'There are exactly $i$ good arguments in favour of H' $(0 \leq i)$. Again, it seems clear that agents can generally be uncertain about the value of $Y$. Many rational agents would surely plead ignorance as to whether or not there exist any undiscovered good argument for the existence of God (and if so, how many). Again, knowledge of the value of $Y$ renders $\mathrm{F}$ independent of $\mathrm{H}$ : If $\mathrm{I}$ know that there are ten good arguments in favour of the existence of God, the fact that I haven't yet found any one of them yet should be irrelevant to my belief in God's existence. Furthermore, if I learn that there are more good arguments for God's existence than I previously thought, that should raise my degree of belief in the existence of God. Finally, the more arguments there are for

\footnotetext{
${ }^{3}$ Actually, this is a slightly simplified version of the theorem proven by DHS. The proof proceeds accordingly.
} 
God's existence, the more likely it is that I find one. (At least the probability that God exists does not go down if more arguments become available.) As before, these considerations give rise to the following basic conditions:

NRF1: $\forall i \geq 0, y_{i}:=P\left(\mathrm{Y}_{\mathrm{i}}\right) \in[0,1)$.

NRF2: $h_{i}:=P\left(\mathrm{H} \mid \mathrm{Y}_{\mathrm{i}}\right)$ are monotonically increasing in $i$.

NRF3: $f_{i}:=P\left(\mathrm{~F} \mid \mathrm{Y}_{\mathrm{i}}\right)$ are monotonically decreasing in $i$.

NRF4: $H$ and $F$ are conditionally independent on $Y$, i.e. $P(\mathrm{H} \mid \mathrm{Y}, \mathrm{F})=$ $P(\mathrm{H} \mid \mathrm{Y})$.

NRF1-4 are structurally near-identical to the conditions imposed on the NAA argument. The only difference is that the $h_{i}$ are now monotonically increasing in $i$. The proof for the following theorem is exactly analogous to that used by DHS for Theorem 1.

Theorem 2 Let $P$ be a probability distribution satisfying the conditions $\mathrm{NRF} 1--4$. Then $\mathrm{F}$ disconfirms $\mathrm{H}$, i.e. $P(\mathrm{H} \mid \mathrm{F})<P(\mathrm{H})$, if there exists a pair $(i, j)$ with $i>j$ such that (i) $y_{i} y_{j}>0$, (ii) $f_{i}>f_{j}$, and (iii) $h_{i}<h_{j}$.

We contend that Theorem 2 constitutes, under certain special circumstances, a full Bayesian vindication of 'no reason for is a reason against' arguments (henceforth 'NRF's') of the type advocated, for example, by Hanson (1971) and mentioned at the beginning of this article. We turn now to clarifying and preemptively defending this claim against a potential objection.

The most pressing criticism of NRF type arguments under our analysis is that they can equally be used against both a hypothesis $\mathrm{H}$ and its negation $\neg \mathrm{H}$. For example, just as one can use an absence of arguments in favour of God's existence to argue for her non-existence, it is equally legitimate to use the absence of arguments for God's non-existence to argue for her existence. And NRF type arguments are only really interesting in cases in which there are no arguments supporting either $\mathrm{H}$ or $\neg \mathrm{H}$. So if they can’t distinguish between $\mathrm{H}$ and $\neg \mathrm{H}$ in these cases, it is hard to see how they could ever be useful.

We agree that this kind of criticism places an important and strong restriction on the applicability of NRF arguments. If an agent considers only the fact that they have not yet encountered a good argument in favour of $\mathrm{H}$ but ignores the fact that they have not yet encountered a good argument 
against $\mathrm{H}$, then they will generally be guilty of selective epistemic vision. They will have reached a conclusion without considering all of the relevant evidence. And indeed, one might contend that this constitutes a fatal flaw in Hanson's (1971) reasoning.

However, it should also be noted that there are particular kinds of reasoning contexts where although the agent does have beliefs about possible arguments in favour of $\mathrm{H}$, they do not have beliefs about possible arguments against H. For example, consider the judge of a murder case in which the suspect has an unverifiable alibi. ${ }^{4}$ The judge would remind the jury that the suspect is innocent until proven guilty. This means that the burden of proof is on the prosecution rather than the defence. Given the unverifiability of the suspect's alibi, the jury is explicitly admonished to ignore the absence of arguments against the suspect's guilt, and concentrate only on the possible arguments in favour of their guilt. In cases like this, where the agent suspends belief about the possible arguments against the truth of $\mathrm{H}$, NRF type arguments can legitimately be used in support of $\mathrm{H}$, but not in support of $\neg \mathrm{H}$.

Returning to the theological example, Hanson writes:

'[A] proof of X's non-existence' usually derives from the fact that there is no good reason for supposing that $\mathrm{X}$ does exist. Since there is no good reason whatever for supposing that green goblins do exist, that fact is normally what is meant by reference to the 'proof' that green goblins do not exist. (Hanson 1971: 311)

Thus, according to Hanson, there is a basic epistemic a-symmetry between positive and negative existential claims. For Hanson, an argument against a negative existential claim is just an observation to the effect that there are no good arguments in favour of the corresponding positive existential claim. If this is right, then an agent who only considers arguments in favour of God's existence really is considering all the relevant evidence, since there are no independent arguments against God's existence. Of course, this is a controversial position relating to complex theological issues concerning, for example, whether God's existence/non-existence is a contingent or analytic matter. But if one buys the conjectured a-symmetry between positive and

\footnotetext{
${ }^{4}$ Suppose for example that the suspect claimed to be at home on the night of the murder, and to have had no contact with anybody all night etc.
} 
negative existential claims, then it looks like Hanson's NRF style arguments can be legitimately applied.

The crucial point is that there do seem to be particular kinds of reasoning scenarios in which agents are permitted (or even required) to only consider possible arguments in favour of the considered hypothesis $\mathrm{H}$, and ignore potential arguments against $\mathrm{H}$. In those special cases, it is possible to give a fully Bayesian justification of the application of NRF style arguments.

\section{References}

Bovens, L. and S. Hartmann (2003). Bayesian Epistemology. Oxford: Oxford University Press.

Dawid, R. (2013). String Theory and the Scientific Method. Cambridge: Cambridge University Press.

Dawid, R., S. Hartmann and J. Sprenger (2015). The No Alternatives Argument. The British Journal for the Philosophy of Science 66(1): $213-234$.

Hanson, N.R. (1971). What I Do Not Believe, and Other Essays. Synthese Library. Dordrecht: Springer.

Howson, C. and P. Urbach (2005). Scientific Reasoning: The Bayesian Approach. Open Court. 